\title{
Salary for Smoking Break (SSB) of Civil Servants (CS) in Japan
}

\author{
Jun Sono, Hajime Ishikawa Sono, Yoshikazu Saito \\ Tobacco-Free Advocacy Japan, Osaka, Japan \\ Email address: \\ jsono@mse.biglobe.ne.jp (J. Sono), pinkpink@msd.biglobe.ne.jp (H. I. Sono)
}

\section{To cite this article:}

Jun Sono, Hajime Ishikawa Sono, Yoshikazu Saito. Salary for Smoking Break (SSB) of Civil Servants (CS) in Japan. Science Journal of Public Health. Vol. 6, No. 2, 2018, pp. 50-54. doi: 10.11648/j.sjph.20180602.13

Received: December 1, 2017; Accepted: December 18, 2017; Published: February 2, 2018

\begin{abstract}
Salary for Smoking Break (SSB) of Civil Servants (CS) of Japan was estimated after a survey of the number of smoking CS during office hours at outdoor Designated Smoking Area (DSA) of two cities, namely Amagasaki and Nishinomiya. The total number of smoking CS during office hours per day at these city main offices was 547 and 400 respectively. The total SSB of CS of two cities combined per year is estimated as more than US\$ 2 million. Approximately, the number of CS all over Japan is 526 times as much as that of two cities combined. Based on this data, total SSB during office hours of CS all over Japan is estimated as more than US\$ 1,052 million. The source of this huge amount of SSB for CS is the tax paid by the people and is not acceptable from tax-payers' point of view. Ban on smoking of CS during office hours is beneficial not only for smoking CS to have the chance to quit or reduce smoking, but also for no smoking colleagues to be free from extra work during the absence of smokers for smoking break and to avoid the risk of the exposure to third-hand smoke from returned smokers. Therefore, ban on smoking of CS during office hours should be welcomed not only by the people, but also by CS regardless of smoking status. This ban could be extended to private work places as a good role model, and protect the health of workers from the risk of active and passive smoking including third-hand smoke. The implementation of this ban may help the better understanding of Japanese people for tobacco de-normalization as the social norm.
\end{abstract}

Keywords: Smoking Ban, Japan, Salary for Smoking Break, Civil Servant, Third-Hand Smoke, De-Normalization, Smoke-Free, WHO FCTC

\section{Introduction}

Japan ratified the World Health Organization's Framework Convention on Tobacco Control (WHO FCTC) in 2004, although the Japanese government proposed "appropriate" and optional measures for final text to accommodate flexibility on key tobacco controls of WHO FCTC [1]. Japan is criticized as one of the lightest market of tobacco in the world [2]. Tobacco control policy of Japan is not yet compliant with WHO FCTC, partly because the publicity of WHO FCTC by the government is limited as the ministry of finance, the important shareholder of Japan Tobacco (JT) Industry, has the strongest power on tobacco policy among ministries. Tobacco de-normalization as the social norm is associated with tobacco control policy of the government [3]. Japan still needs to amend to strengthen Health Promotion Law of 2005, which stipulates that the manager of public places need to protect people from exposure to second-hand smoking. This needs to be strengthened by inclusion of a penalty clause. Therefore, Health Promotion Law should ensure that public places and indoor work places should be declared $100 \%$ smoke-free without exemption as mandated by Article 8 Guideline of WHO FCTC, and should be implemented for government offices at first in Japan.

People are always keen on the working attitude and behavior of CS, especially from tax payers' point of view, because tax paid by people is the source of the salary of CS. Therefore, it is important to estimate the SSB of CS for the better understanding of people to introduce the ban of smoking of CS during work time. The number of smoking CS during office hours at two city main offices of Amagasaki and Nishinomiya was surveyed and SSB of two cities was calculated. "Figure 1" shows the location of two cities in Hyogo prefecture of Japan. The number of CS of Amagasaki is 3,196 and that of Nishinomiya is 3,121 . The number of CS all over Japan is 3,323,000 and approximately 526 times as much as two cities combined. 


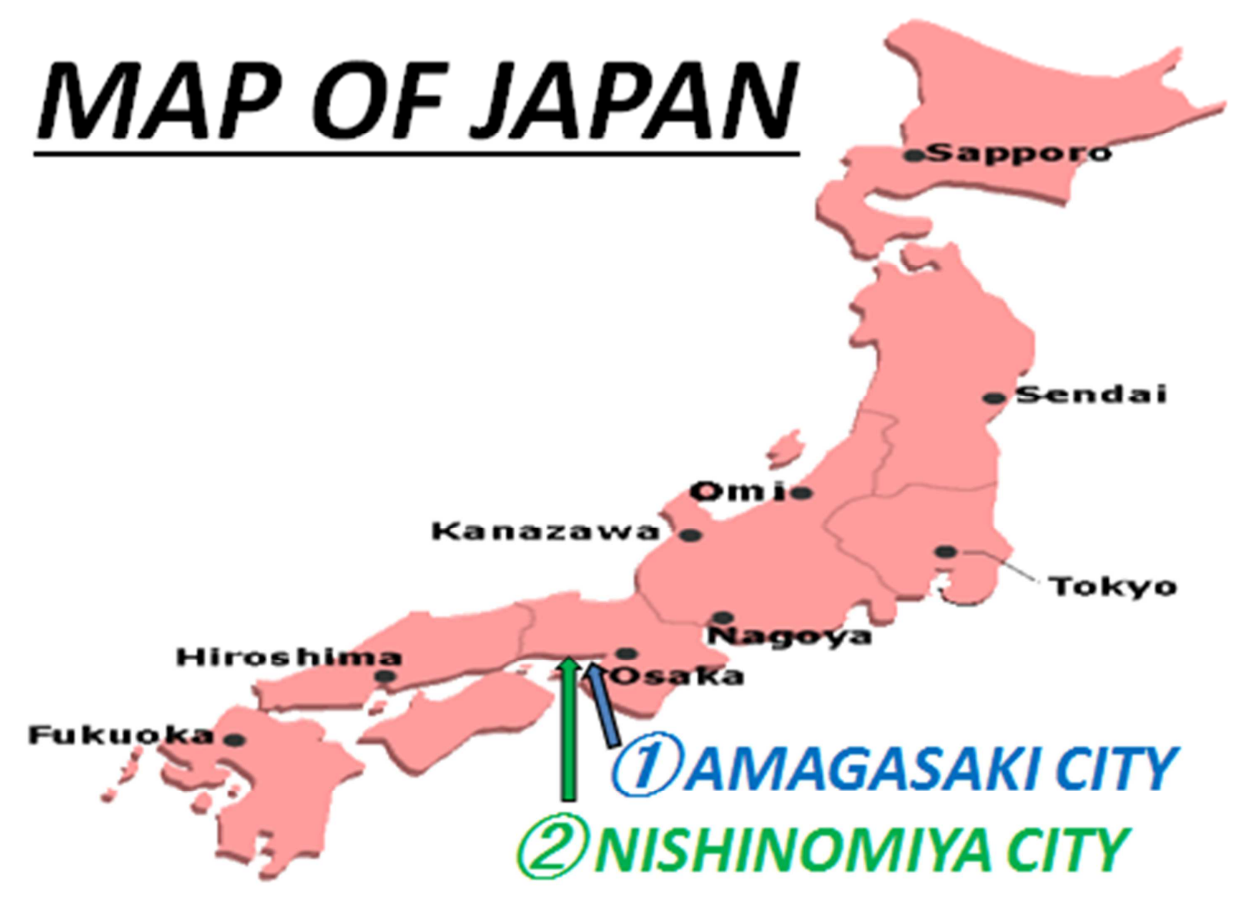

Figure 1. Map of Japan and the location of two cities, Amagasaki and Nishinomiya.

\section{Method and Materials}

\subsection{Survey of Smoking CS During Office Hours at Amagasaki City Main Office}

On the $23^{\text {rd }}$ of July 2015 , the number of smoking CS during office hours, from 9:00 to 17:30 was counted. The only Designated Smoking Area (DSA) of CS is located at the corner of basement parking area of cars for public use owned by the city. The number of CS who smoked at the DSA was monitored from a distant place, avoiding the exposure to second-hand smoke, as shown as "Figure 2". Because this DSA in not available for the citizens, all the smokers are assured to be CS. The total number of smoking CS was 547, excluding 97 during lunch time from 12:00 to13:00.

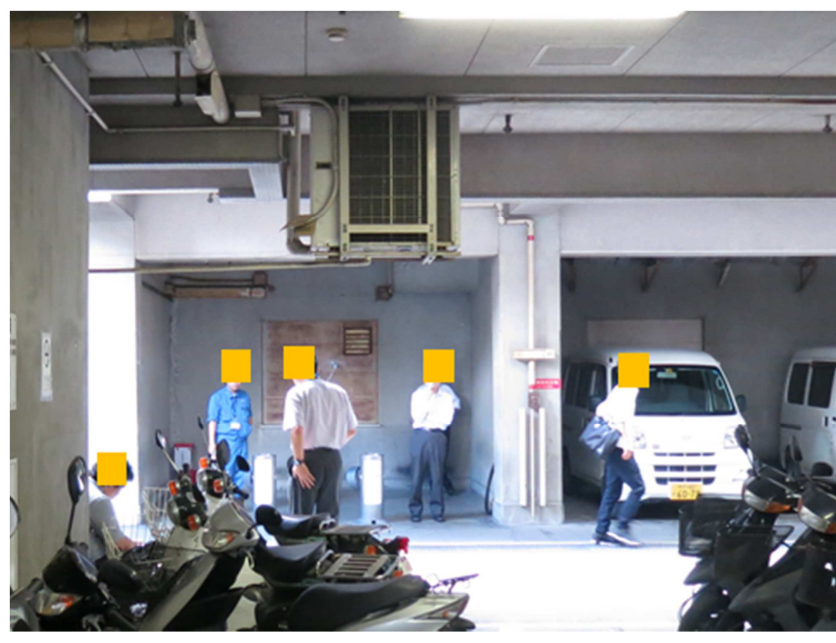

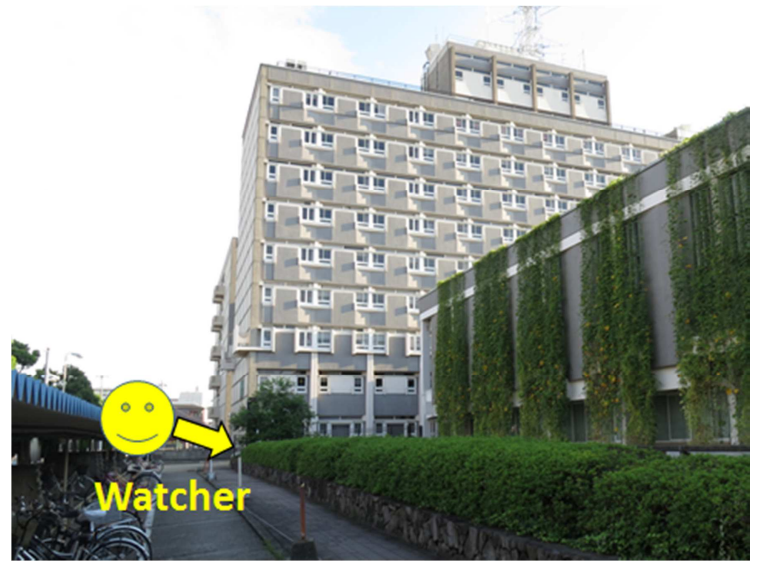

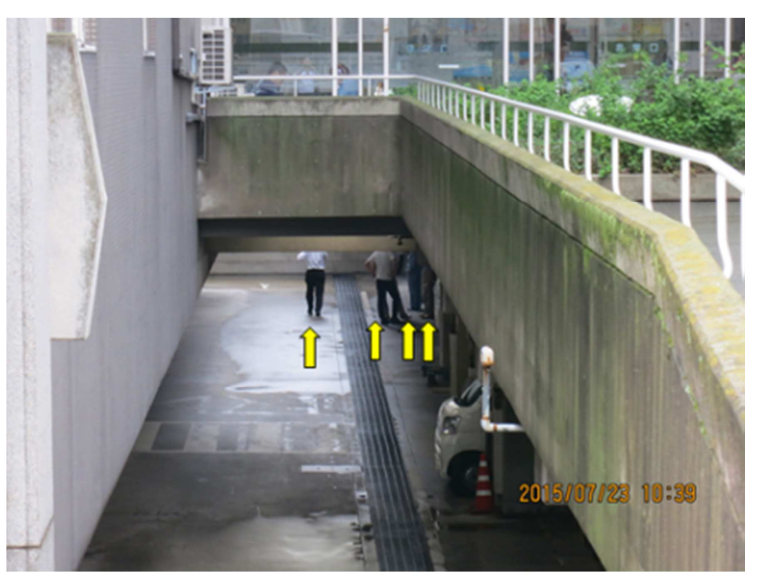

Figure 2. Basement Designated Smoking Area (DSA), exclusively for Civil Servants (left), Amagasaki City Main Office and the watching point (middle), and the view of DSA and four smokers(arrows) from the watching point (right). 


\subsection{Estimation of SSB of CS of Amagasaki City}

Assumption and calculation of SSB of Amagasaki CS are as follows (Table1). One Smoking Break Time (\#1) is assumed as 10 minutes including the time they walk to and from outdoor DSA. Total number of CS smoking during office hours at main office per day (\#2) was 547. Total smoking break time per day (\#3) is 5,470 minutes (10min. X 547=91.2 hours). Actual work day of CS per year is 216 days, which is equivalent to 1728 hours (\#4), because work time is 8 hours per day. The average salary of an Amagasaki City CS (\#5) is US\$ 64,350 per year, provided 100
JP Yen equals to 1US\$. Therefore, Actual Salary per Work Hour (ASPWH, \#6) is US\$37.24 (=US\$ 64,350/ 1728 hours). Total SSB per Day (TSSBPD, \#7) of the city main office is calculated as US\$ 3,396.3 (=US\$ 37.24 X 91.2 hours) and annual SSB per year (\#8) becomes US\$ 733,601 (= TSSBPD X 216 days). In Amagasaki, there are more than 10 branch offices, including the department of water service and fire station in addition to the city main office and annual SSB of CS of all over Amagasaki (\#9) is estimated as more than US\$ 1 million.

Table 1. Assumption and calculation of Salary for Smoking Break of Amagasaki City Civil Servants.

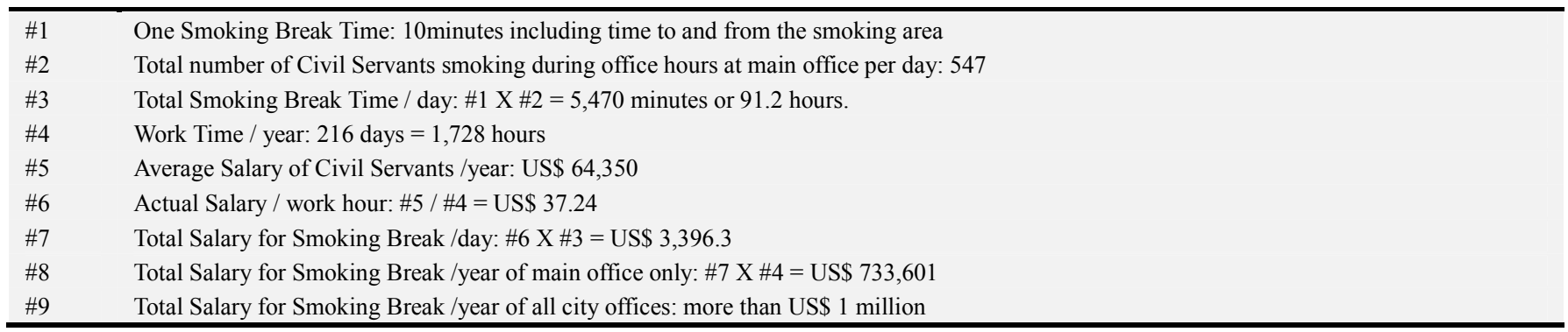

\subsection{Survey of Smoking CS of Nishinomiya City Main Office and Estimation of SSB of CS in Nishinomiya}

On the $25^{\text {th }}$ May 2015, similar survey as Amagasaki was conducted at the outdoor DSA of CS of Nishinomiya City Main Office. The total number of smoking CS during office hours was estimated at 400, excluding lunch time. Similar assumption and calculation revealed that annual SSB was US\$.570,240. In Nishinomiya, there are more than 10 branch offices including the department of water service and fire station in addition to the city main office and annual SSB of CS all over Nishinomiya is estimated more than US\$ 1 million.

\subsection{Assumption and Calculation of Annual SSB of CS of All over Japan}

Table 2. Assumption and calculation of annual Salary for Smoking Break of Civil Servants all over Japan.

\begin{tabular}{ll}
\hline$\# 1$ & Total number of Civil Servants of Amagasaki City: 3,196 \\
$\# 2$ & Total number of Civil Servants of Nishinomiya City: 3,121 \\
$\# 3$ & Total number of Civil Servants of Both Cities combined: $\# 1+\# 2=6,317$ \\
$\# 4$ & Salary for Smoking Break of Both Cities combined per year: more than US\$ 2 million \\
$\# 5$ & Total number of Civil Servants of Local Governments in Japan: $2,739,000$ \\
$\# 6$ & Total number of Civil Servants of Central Government in Japan: 584,000 \\
$\# 7$ & Total number of Civil Servants all over Japan: \#5 + \#6 =3,323,000 \\
$\# 9$ & Total number of Civil Servants all over Japan compared to Both Cities combined: more than 526 times $=\# 7 / \# 3$ \\
\hline
\end{tabular}

The estimation of annual SSB of CS all over Japan is shown as follows. (Table 2) The number of CS of Amagasaki (\#1) is 3,196, and that of Nishinomiya (\#2) is 3,121. The total number of CS of both cities combined (\#3) is 6,317. The annual SSB of both cities combined (\#4) is estimated as more than US\$ 2 million, because that of two cities is more than US\$ 1 million respectively. The number of CS of Central Government (\#5) is 584,000, and that of all Local Governments (\#6) is 2,739,000. Therefore, the total number of CS all over Japan (\#7) is 3,323,000, and is approximately 526 times as much as two cities combined (\#8). [8] Therefore, annual SSB for CS of all over Japan (\#9) is 526 times of that of two cities combined and estimated as more than US\$ 1,052 million (= \#4 X \#8).

\section{Discussion and Consideration}

According to Australian reports, work place smoking bans have not only reduced work-day cigarette consumption, but also been associated with going outside to smoke during working hours [4] [5] [6]. Even in Japan, indoor work place smoking bans become a social norm, especially at most of public offices. Subsequently, leaving work to smoke outside during office hours becomes problematic. This study focused on SSB from tax payers' point of view, taking deep concern of the people on the working attitude and behavior of CS into account. This study also aimed at the beneficial effect on the health of smoking CS to promote the motivation to quit smoking. "Smoke-free grounds" worksite policy to prohibit 
leaving work to smoke outside during office hours gained higher quit rates after the ban [7]. Unofficial Smoking Break during office hours is considered not only a waste of work time, but also a burden and a risk for no smoking colleagues. They need to take over the responsibilities of those who frequently go out to smoke and suffer from exposure to third-hand smoke from returned smokers. Recent studies disclosed the danger of exposure to third-hand smoke on biomarker level and on clinical basis [8] [9] [10]. In addition, the unofficial smoking break during work time of CS is against the duties and obligations stipulated by public service act.

There are five problems on the assumption of this study. Firstly, one smoking break time assumed at 10 minutes might be shorter compared to the real time spent for smoking at DSA outdoors. Recently, a lot of smokers manipulate the smart phone at DSA. On Nishinomiya survey, there was a CS with his smart phone, who spent 45 minutes in the morning and in the afternoon respectively at DSA. One smoking break time is also influenced by the distance between indoor work place and outdoor DSA. Secondly, we counted the number of smoking CS in both City Main Offices only and assumed SSB of CS of all two cities, including other more than ten branches respectively. Thirdly, there is fluctuation of the exchange rate of 100 Japanese Yen (JP Yen) to US\$ 1. Recent exchange rate of currency is around $111 \mathrm{JP}$ Yen for US\$1. Fourthly, as regards to the average annual salaries of CS, there is a difference among local governments and central government. In Japan, Laspeyres Index is used to assess the average annual salary of CS of local governments compared to that of central government, which is provided as $100 \%$. Laspeyres Index of Amagasaki is $97.2 \%$ and that of Nishinomiya is $101.6 \%$. The difference of the salary of both cities from central government is thought to be negligible. Fifthly, smoking prevalence of CS may differ among central and local governments, but this slight difference could be negligible

On March 2016, a member of Yokohama City Assembly in Japan estimated annual SSB of Yokohama CS at around US\$.15,400,000. The number of CS of all over Japan $(3,323,000)$ is approximately 165 times of Yokohama $(20,112)$. According to this estimation, annual SSB of CS all over Japan is estimated at US\$ 2,541 million which is more than twice of our estimation. This might be partly due to the higher Laspeyres Index of Yokohama (103.7\%) compared to those of both cities, Amagasaki $97.2 \%$ and Nishinomiya $101.6 \%$ respectively. Therefore, the amount of annual SSB of CS is enormous regardless of the method of estimation.

Japan is the host country of the 2020 Tokyo Olympics and Paralympics. WHO and International Olympic Committee have requested the host cities should observe a "Smoke-free" environment. However, even now the move towards "Smoke-free public places and indoor work place" remains sluggish in Japan. With regard to smoke-free policy, there is always a strong opposition from hospitality industries, such as restaurants, coffee shops, bars and hotels, supported by the tobacco industry. One of their arguments is that the central and local governments should take initiative and implement a smoke-free public office first, then the hospitality industry will gradually introduce smoke-free environment.

It is important for Japanese government to ban on smoking of CS during office hours and extend this good role model to the private work places. This ban could be extended to private work places as a good role model, and protect the health of workers from the risk of active and passive smoking including third-hand smoke. The implementation of this ban may help the better understanding of Japanese people for tobacco de-normalization as the social norm.

\section{Conclusion}

SSB of CS in Japan is estimated at more than US\$ 1,052 million, which is not acceptable by all means from tax payers' point of view. Banning smoking during office hours is a good motivation for smokers to eventually quit smoking, thereby improving their work productivity and improving their health condition. No smoking colleagues can be free from the extra work during the absence of smokers for smoking break and avoid the risk of exposure to third-hand smoke. This is a "win, win" proposal, which is beneficial for not only tax-payers, but also CS regardless of their smoking status. In addition, ban on smoking of CS during office hours should have the good influence to private work places and promote tobacco de-normalization as the social norm in Japan.

\section{Acknowledgements}

The authors thank Dr. Domilyn Villarreiz for her linguistic assistance and advice.

\section{References}

[1] M. Assunta and S. Chapman (2006) Health treaty dilution: a case study of Japan's influence on the language of the WHO Framework Convention on Tobacco Control. J Epidemiol Community Health 60(9), 751-756.

[2] M. Assunta and S. Chapman (2008) The lightest Market in the World: Light and Mild Cigarettes in Japan. Nicotine \&Tobacco Research 10(5), 803-810.

[3] D. Hammond, G. Fong, M. Zanna, J. Thrasher, R. Borland (2006) Tobacco denormalization and industry beliefs among smokers from four countries. Am J Prev Med 31(3), 225-32.

[4] R. Borland, N. Owen, B. Hocking(1991) Changes in smoking behavior after a total workplace smoking ban. Aust J Public Health 15(2), 130-134.

[5] R. Borland, and N. Owen (1995) Need to smoke in the context of workplace smoking bans. Prev Med 24(1), 56-60.

[6] R. Borland, M. Cappiello, N. Owen (1997) Leaving work to smoke. Addiction 92(10) 1361-1368.

[7] O. Onisubi, S. Sinha, E. Rovner, M. Perez-Lugo, N. Jain, K. Demissie, M. Goldman(2004) Efficacy of tobacco dependence treatment in the context of a "smoke-free grounds" worksite policy: a case study. Am J Ind Med 46(2), 180-187. 
[8] C. Chen, S. Huang, C. Yang, K. Tang, D. Yao(2016) Detection of third-hand smoke on clothing Fibers with a surface acoustic wave gas sensor. Biomicrofluids 10(1):011907.

[9] S. Dhall, R. Alamat, A. Castro, A. Sarker, J. Mao, A. Chan, B. Hang, M. Martin-Green(2016) Tobacco toxins deposited on surface (third hand smoke) impair wound healing. Clin Sci(Lond)130(14), 1269-1284.
[10] N. Adhami, Y. Chen, M. Martins-Green(2017) Biomarkers of disease can be detected in mice asearlyas 4 weeks after initiation of exposure to third-hand smoke levels equivalent to those found in homes of smokers. Clin Sci(Lond) 131(19), 2409-2426. 